\title{
BALANCED SCORECARD NA PRÁTICA DA GESTÃo DE UM LABORATÓRIO ACADÊMICO
}

\section{RESUMO}

O Balanced Scorecard (BSC), utilizado em todo seu potencial, é um sistema de gestão estratégica essencialmente utilizado por empresas. Entretanto, com adaptações, pode ser utilizado por Organizações Sem Fins Lucrativos (OSFL). Seu uso, neste caso, ainda precisa ser mais bem investigado. O artigo relata a experiência e lições aprendidas com o BSC em um laboratório acadêmico durante dezesseis meses. O processo incluiu planejamento inicial, três rodadas de medição de desempenho e revisão do planejamento. O principal resultado do processo foi o fortalecimento da identidade do laboratório. O BSC, com algumas alterações em relação ao modelo usado por empresas, mostrou-se adequado para definição coletiva da estratégia, fortalecimento da identidade do grupo envolvido, medição de desempenho e comunicação de resultados sem, no entanto, ter alcançado a maturidade de um sistema de gestão estratégica durante o período da experiência.

Palavras-chave: Aprendizado Organizacional; Gestão Estratégica; Organização sem Fins Lucrativos.

\section{BALANCED SCORECARD IN THE PRACTICE OF MANAGEMENT AT AN ACADEMIC LABORATORY}

\section{ABSTRACT}

The Balanced Scorecard (BSC), when it reaches its maximum potential, is a strategic management system mainly used by companies. However, with some adjustments, it can be used by Non-profit Organizations (NPO). Its use, in this case, needs to be further investigated. The article reports the experience and lessons learned from using BSC in an academic lab for sixteen months. The process included initial planning, three rounds of performance measurement and a final planning review. Entrepreneurial BSC, with some adjustments, was adequate for strengthening laboratory's identity, for performance measurement and results reporting, although not reaching, during the experiment period, the maturity of a strategic management system.

Keywords: Organizational Learning; Strategic Management; Non-Profit Organization. 


\section{BALANCED SCORECARD EN LA PRÁCTICA DE GESTIÓN DE UN LABORATORIO ACADÉMICO}

\section{RESUMEN}

El Balanced Scorecard (BSC), que se utiliza en todo su potencial, es un sistema de gestión estratégica utilizado principalmente por las empresas. Sin embargo, con adaptaciones, puede ser utilizado por organizaciones sin fines de lucro (NPO). Su uso en este caso, la necesidad de mayor investigación. El artículo reporta la experiencia y lecciones aprendidas del BSC en un laboratorio académico durante dieciséis meses. El proceso incluyó la planificación inicial, la medición del desempeño de tres rondas y revisión de planificación. El principal resultado del proceso fue el de fortalecer la identidad del laboratorio. El BSC, con algunos cambios respecto al modelo que utilizan las empresas, era adecuado para la definición colectiva de la estrategia, el fortalecimiento de la identidad del grupo involucrado, la medición del desempeño y los resultados de informes, sin embargo, después de haber alcanzado la madurez de un sistema gestión estratégica durante el período del experimento.

Palabras-clave: Aprendizaje Organizacional; Gestión Estratégica; Organización sin Fines de Lucro.

Miriam Magdala Pinto ${ }^{1}$ Bruno Mannato Angius ${ }^{2}$

\footnotetext{
${ }^{1}$ Doutora em Engenharia de Produção pela Pontifícia Universidade Católica do Rio de Janeiro - PUC/RJ. Professora da Universidade Federal do Espírito Santo - UFES. Brasil. E-mail: miriammagdalapinto@gmail.com

2 Graduado em Engenharia de Produção pela Universidade Federal do Espírito Santo - UFES. Brasil. E-mail: bmannato@gmail.com
} 
Balanced Scorecard na Prática da Gestão de um Laboratório Acadêmico

\section{INTRODUÇÃO}

A utilização do BSC para apoiar a gestão de Organizações Sem Fins Lucrativos (OSFL) ainda é limitada e precisa ser mais bem investigada (Hoque, 2013). Por outro lado, cada vez mais, organizações desse tipo se vêm impelidas a profissionalizar sua gestão. Organizações da administração pública são chamadas a abandonar modelos de gestão burocráticos e substituí-los por modelos centrados em resultados e eficiência, no caso, no atendimento aos cidadãos ao lado do controle orçamentário (Mendes, Santos, Perna, \& Teixeira, 2012). Os mesmos autores afirmam que a introdução e uso de ferramentas oriundas do setor privado com adaptações para o setor tem sido bem sucedidos.

Este artigo contribui para o entendimento do uso e do valor do BSC no apoio à gestão de laboratórios acadêmicos que são geralmente OSFL por meio de estudo de caso único: o do Laboratório de Tecnologias de Apoio a Redes de Colaboração da Universidade Federal do Espirito Santo (LabTAR/UFES), laboratório multidisciplinar do qual participam professores, estudantes e bolsistas ligados aos Departamentos de Informática, Desenho Industrial e Engenharia de Produção da universidade. Nele são desenvolvidos projetos de pesquisa, de extensão e de inovação. Ele é a organização central do Living Lab Habitat, uma rede de colaboração que integra academia, empresas, governo e ONGs com o propósito de inovar visando à melhoria de condições habitacionais de populações de baixa renda.

\section{REFERENCIAL TEÓRICO}

O Planejamento Estratégico é um processo sistemático que compreende análise e síntese. Analisa-se primeiramente a organização e seu ambiente de modo a subsidiar o processo de síntese subsequente no qual a organização define marcos que guiarão suas decisões e ações futuras. Tais processos foram amplamente discutidos nas décadas de 1960 e 1970 (Ansoff, 1965; Mintzberg, 1973, 1990).

A separação entre a etapa de Planejamento Estratégico formal e racional feita inicialmente e a implantação da estratégia definida posteriormente em ambientes turbulentos foi motivo de debates acadêmicos intensos durante as décadas de 1980 e 1990 (Idenburg, 1993). A necessidade de integrar decisões estratégicas com ações no nível operacional das organizações mostrava-se imperativa.

Uma possibilidade de integração de estratégia e ações surgiu com o BSC. Inicialmente proposto como sistema para medição do desempenho organizacional (Kaplan \& Norton, 1992), passou a ser utilizado para identificar processos organizacionais críticos a ser monitorados (Kaplan \& Norton, 1993) e, finalmente, como sistema de gestão estratégica (Kaplan \& Norton, 1996a), um conjunto de processos que permite à organização estabelecer, controlar e monitorar a execução de sua estratégia, de modo contínuo.

O BSC, enquanto sistema de gestão estratégica supre o gap entre a Missão e a Visão da organização, as iniciativas e orçamentos necessários para torná-las realidade ao mesmo tempo em que permite o monitoramento sistemático da evolução da organização rumo à sua visão e o aprendizado estratégico. Enquanto sistema de gestão estratégica, o BSC viabiliza: i) esclarecer a missão e a visão da organização; ii) comunicar e associar objetivos e medidas estratégicas, iii) planejar, estabelecer metas e alinhar iniciativas estratégicas e iv) melhorar o feedback e o aprendizado estratégico (Kaplan \& Norton, 1996a).

A metodologia do BSC prevê a identificação de Objetivos Estratégicos (OE) dentro de cada uma das quatro perspectivas, ligando-se uns aos outros em relações de causa e efeito que, dispostos em um diagrama dentro das quatro perspectivas, formam o Mapa Estratégico (ME), que explicita a estratégia da organização (Niven, 2007).

O BSC foi desenvolvido para, e tem sido utilizado principalmente por empresas. No entanto, os próprios criadores do BSC propõem seu uso por OSFL. Para adaptar o BSC para OSFL é necessário que a organização meça seu sucesso pelo grau em que atende às necessidades de seus principais stakeholders (Kaplan \& Norton, 1996a) e alcança sua visão (Niven, 2007). Kaplan e Norton (1996a) orientam ainda que OSFL devem colocar sua missão no topo do ME, uma vez que elas não buscam o lucro como demonstrativo de sucesso, mas atingir um propósito que busca a melhoria de algum aspecto da sociedade. A missão deve ser objetivamente definida, evitando-se conceitos abstratos, para que possa ser desdobrada em OE.

\section{METODOLOGIA}

O Planejamento Estratégico para o LabTAR foi baseado em Oliveira (2011) e Kaplan \& Norton (1996). O período total da experiência relatada neste artigo é de dezesseis meses, indo de setembro de 2012 a fevereiro de 2014. Durante este período foram realizadas uma rodada inicial de Planejamento Estratégico, três avaliações trimestrais do desempenho, uma rodada de revisão 
do Planejamento Estratégico e uma reunião de análise crítica fechando um ciclo de gestão do laboratório.

A rodada inicial de planejamento foi feita em cinco encontros, de aproximadamente três horas de duração cada, com participação parcial dos treze membros do laboratório, pois nem todos compareceram a todos os encontros, onde foram apresentados conceitos, realizadas atividades em grupos e de formação de consenso e definidas atividades para serem feitas em casa para subsidiar os encontros subsequentes. A Figura 1 representa o fluxo de atividades realizadas.

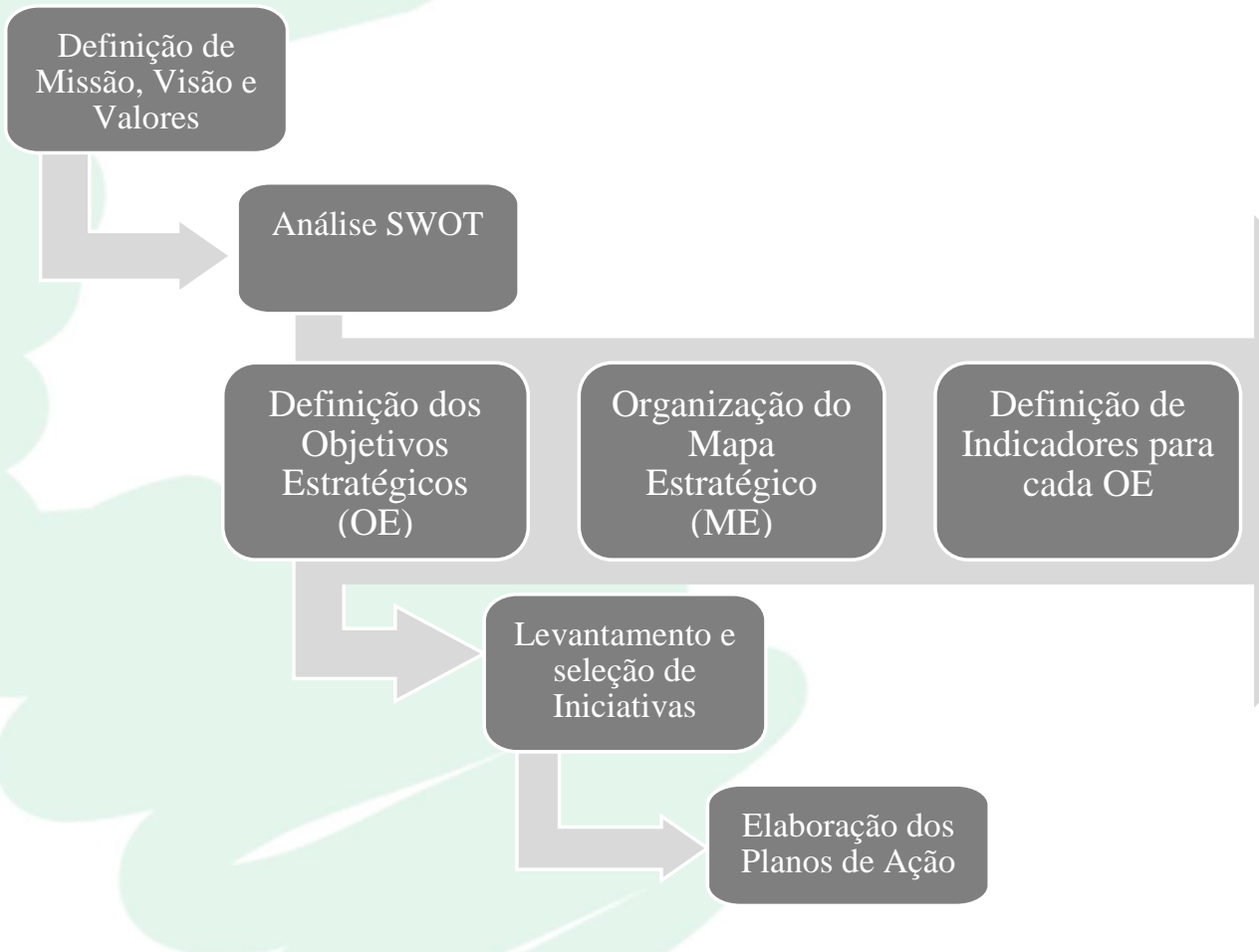

Figura 1 - Representação esquemática do fluxo de atividades realizado

Ao longo dos encontros, os membros explicitaram Missão, Visão e Valores da organização, foi feita Análise SWOT, e iniciou-se a construção do BSC. O ME foi construído com a definição de $\mathrm{OE}$ dentro das Perspectivas de Geração de Valor, Stakeholders, Processos Internos e Colaboradores. Em seguida, foram selecionados Indicadores para medição de desempenho e definidas Metas para cada Indicador. Finalmente, foram definidas Iniciativas, desdobradas em Planos de Ação, que favorecessem o atingimento de cada OE. As três avaliações trimestrais de desempenho foram feitas utilizando formulários online $\mathrm{e}$ avaliações pessoais. $\mathrm{O}$ resultado de desempenho em cada Indicador era convertido de acordo com escala de cinco cores. A revisão do PE foi feita quinze meses após o início do processo quando apenas quatro dos treze membros do LabTAR que participaram do $\mathrm{PE}$ inicial permaneciam no laboratório, ou seja, 30\% deles. Apenas eles participaram da reunião de análise crítica final para avaliação de toda a experiência.

\section{RESULTADOS OBTIDOS E ANÁLISE}

Nesta seção são apresentados e discutidos os resultados obtidos pelo LabTAR durante o período de dezesseis meses em que usou o Planejamento Estratégico com BSC em sua rotina de trabalho para apoio à gestão.

\section{Identidade}

A identidade de uma organização é geralmente expressa pelas afirmações de sua Missão, Visão e Valores. O LabTAR possuía definição de Missão, Visão e Valores anterior ao processo de planejamento descrito neste artigo. Eles haviam sido definidos em 2011 por meio de redação colaborativa não haviam sido incorporados à sua cultura pois apenas os valores estabelecidos em 2011 foram lembrados na rodada inicial de PE. 
Balanced Scorecard na Prática da Gestão de um Laboratório Acadêmico

No Quadro 1 são mostradas as três versões de

Missão, Visão e Valores para o LabTAR.

\begin{tabular}{|c|c|}
\hline \multicolumn{2}{|r|}{ Missão do LabTAR } \\
\hline Versão 2011 & $\begin{array}{l}\text { Oferecer apoio tecnológico e expertise no desenvolvimento de projetos em redes de } \\
\text { colaboração. }\end{array}$ \\
\hline $\begin{array}{l}\text { Versão } \\
2012 / 2013\end{array}$ & $\begin{array}{l}\text { Fomentar a criação e o desenvolvimento de redes de colaboração envolvendo os diversos } \\
\text { segmentos da sociedade e prover tecnologias de apoio aos processos de trabalho nessas } \\
\text { redes. }\end{array}$ \\
\hline Versão 2014 & $\begin{array}{l}\text { Fomentar a criação e o desenvolvimento de redes de colaboração para inovação } \\
\text { envolvendo os diversos segmentos da sociedade e prover tecnologias de apoio aos } \\
\text { processos de trabalho nessas redes. }\end{array}$ \\
\hline \multicolumn{2}{|r|}{ Visão do LabTAR } \\
\hline Versão 2011 & $\begin{array}{l}\text { Ser reconhecido internacionalmente como polo gerador e difusor de conhecimento em } \\
\text { gestão de redes de colaboração. }\end{array}$ \\
\hline $\begin{array}{l}\text { Versão } \\
2012 / 2013\end{array}$ & $\begin{array}{l}\text { Ser reconhecido como agente de integração, transformação e causador de impacto positivo } \\
\text { na sociedade e ser referência nas áreas de competência do laboratório. }\end{array}$ \\
\hline Versão 2014 & $\begin{array}{l}\text { Ser referência local, nacional e internacional como agente de integração, transformação e } \\
\text { causador de impacto positivo na sociedade. }\end{array}$ \\
\hline \multicolumn{2}{|r|}{ Valores do LabTAR } \\
\hline $\begin{array}{l}\text { Versões } 2011 \text { e } \\
2012 / 2013\end{array}$ & $\begin{array}{l}\text { Colaboração, transdisciplinaridade, compartilhamento, excelência e humanização dos } \\
\text { relacionamentos. }\end{array}$ \\
\hline Versão 2014 & $\begin{array}{l}\text { Colaboração, transdisciplinaridade, compartilhamento, excelência, humanização dos } \\
\text { relacionamentos e transparência. }\end{array}$ \\
\hline
\end{tabular}

Quadro 1 - Missão, Visão e Valores do LabTAR nas versões 2011, 2012 e 2014.

Comparando-se as versões, observa-se que, na ocasião do PE inicial, em 2012, restavam dúvidas sobre a identidade do laboratório. A elaboração da Missão e da Visão de forma colaborativa em 2012 favoreceu sua aceitação pelo grupo, pois, na revisão de 2014, as mesmas praticamente não sofreram alterações. Essa estabilidade deu-se apesar de apenas $30 \%$ dos membros do grupo terem participado dos dois momentos de planejamento.

A Análise SWOT foi utilizada para nivelar o conhecimento dos pontos fortes e fracos do laboratório e para explicitar elementos do ambiente externo que podem afetá-lo. Essa análise subsidia a construção do $\mathrm{ME}$, pois a definição dos $\mathrm{OE}$ considera o ambiente da organização para sua formulação.

\section{O Balanced Scorecard}

A Missão, a Visão, os Valores e Análise SWOT formam o arcabouço estrutural da estratégia. Seu desdobramento até o nível operacional foi feito com o Balanced Scorecard BSC.
Como o LabTAR é uma OSFL, a Perspectiva Financeira não é o objetivo final da organização, e foi substituída pela Perspectiva de Geração de Valor. Como o LabTAR não possui clientes diretos, a Perspectiva dos Clientes foi substituída pela dos Stakeholders, englobando as partes interessadas no sucesso do LabTAR. A Perspectiva de Processos Internos foi mantida e a de Crescimento \& Aprendizagem foi renomeada de Colaboradores.

\section{Objetivos Estratégicos}

$\mathrm{Na}$ rodada inicial do Planejamento Estratégico, a definição dos $\mathrm{OE}$ baseou-se na proposta de Kaplan \& Norton (1996a), a saber, os OE propostos para a Perspectiva de Geração de Valor foram extraídos da Visão definida para o LabTAR: 1) Ser agente de integração, transformação e impacto positivo na sociedade e 2) Ser referência em pesquisa nas suas áreas de competência.

Os OE selecionados foram ligeiramente modificados ao longo do processo de monitoramento do desempenho do LabTAR. A 
Balanced Scorecard na Prática da Gestão de um Laboratório Acadêmico

Figura 2 apresenta as versões dos Mapas Estratégicos do LabTAR inicial e revisado.

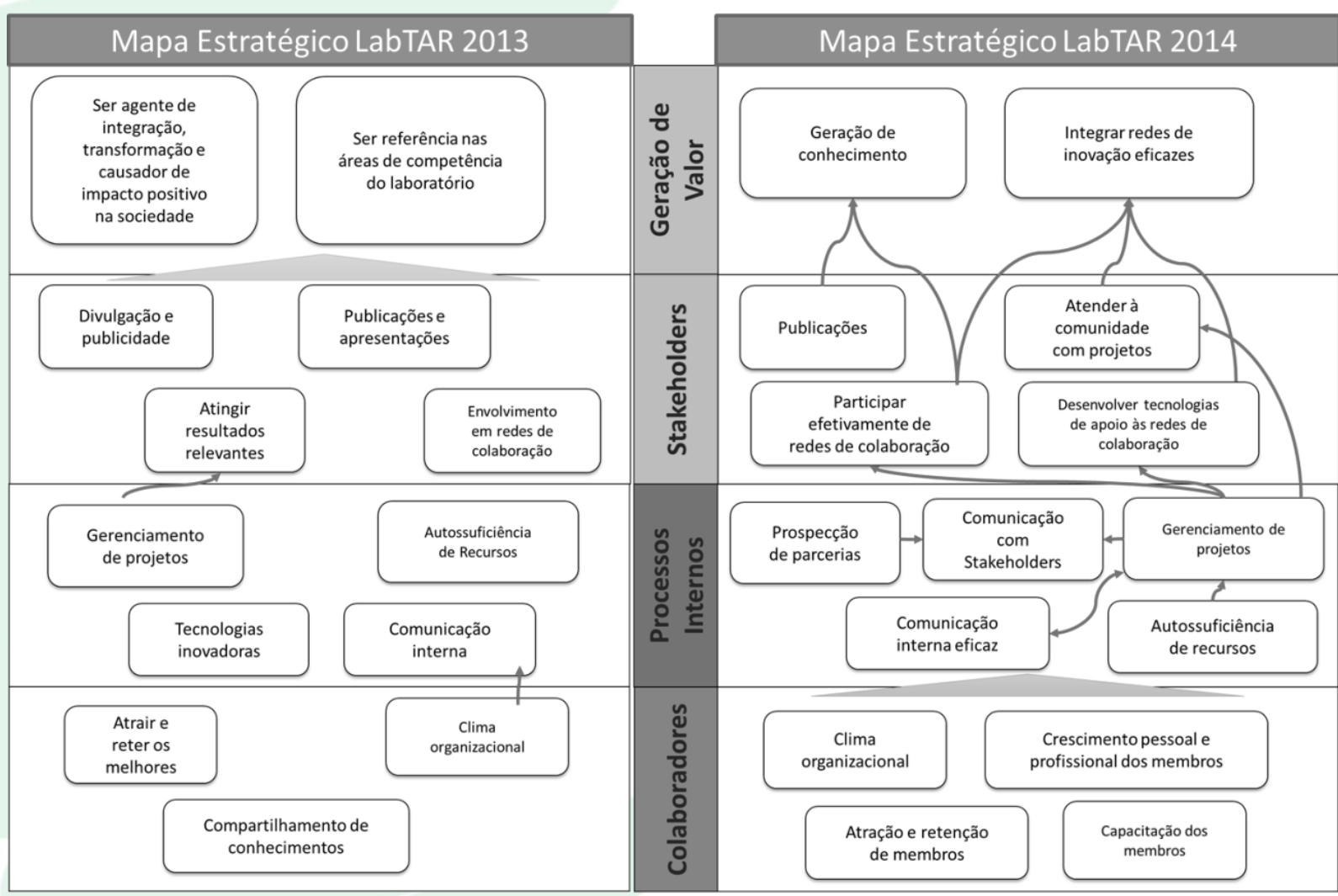

Figura 2 - Versões 2012/2013 e 2014 do Mapa Estratégico do LabTAR

\section{Definição de Indicadores e metas}

Os indicadores foram definidos colaborativamente pelos membros do laboratório a partir de sugestões individuais utilizando-se bom senso e a experiência anterior dos membros. A lista dos indicadores para cada $\mathrm{OE} e$ as metas estabelecidas encontra-se no Quadro 2.

Durante as rodadas de avaliação, indicadores e metas foram revistos apenas pela equipe responsável pelo BSC que observou incoerências entre indicadores e metas propostos. Como durante as rodadas de avaliação e monitoramento, o desempenho do laboratório em cada OE, representado em cores no ME, foi o único feedback levado para todo o grupo, o aprendizado sobre indicadores, metas, suas interrelações e com os OE não foi geral. Esta deficiência no processo só foi detectada na reunião de análise crítica final.

\section{Iniciativas e Planos de Ação}

Definida a identidade do LabTAR e elaborado seu ME com Indicadores e Metas, o passo seguinte foi a identificação de Iniciativas que permitissem atingir tais metas. As Iniciativas foram sugeridas individualmente e selecionadas pelo grupo também com base no bom senso $\mathrm{e}$ experiências anteriores. Para cada Iniciativa foi designado um responsável pela elaboração de um Plano de Ação, formação de equipe e execução do plano. O índice de sucesso na conversão de Iniciativas em Planos de Ação elaborados e executados foi muito baixo. De quinze Iniciativas propostas, apenas sete planos de ação foram elaborados e destes, apenas quatro realizados. A reunião de análise crítica revelou que este insucesso deveu-se à falta de cobrança e alta rotatividade dos membros do LabTAR no período. Esta alta rotatividade foi prejudicial de duas maneiras: baixo interesse ou comprometimento daqueles que estavam deixando o LabTAR em elaborar planos que não ficariam sob sua responsabilidade e devido a descontinuidade de um responsável por uma Iniciativa.

Esta constatação fez com que na revisão do Planejamento Estratégico, ênfase fosse dada a 
Balanced Scorecard na Prática da Gestão de um Laboratório Acadêmico

atribuição de responsáveis e elaboração dos Planos de Ação para cada Iniciativa selecionada.

\section{Monitoramento}

Cada compilação do BSC proporcionou, para a equipe envolvida com a medição, aprendizado quanto à adequação de OE, Indicadores e Metas e sugestões de melhorias para o BSC. No entanto, os demais membros do LabTAR apenas viam a compilação dos dados do BSC traduzida no ME colorido em reunião destinada a este fim. $\mathrm{O} \mathrm{ME}$, a partir da apresentação oral, ficava afixado na parede do laboratório até a atualização seguinte.

O BSC ciclo 2012/2013 com os resultados

de desempenho é apresentado no Quadro 2. O BSC ciclo 2014 com indicadores e iniciativas é apresentado no Quadro 3. 
Balanced Scorecard na Prática da Gestão de um Laboratório Acadêmico

\begin{tabular}{|c|c|c|c|c|c|c|c|c|c|c|c|c|}
\hline \multirow{2}{*}{ Perspectiva } & \multirow{2}{*}{ Objetivo Estratégico } & \multicolumn{2}{|l|}{ Indicador } & \multicolumn{3}{|c|}{ Metas } & \multicolumn{3}{|c|}{ Medição } & \multicolumn{3}{|c|}{ Percentual } \\
\hline & & $13 T 3$ & $13 \mathrm{T4}$ & $13 T 2$ & $13 T 3$ & $13 T 4$ & $13 \mathrm{T2}$ & $13 \mathrm{T3}$ & $13 \mathrm{~T} 4$ & $13 T 2$ & $13 T 3$ & $13 \mathrm{~T} 4$ \\
\hline \multirow{3}{*}{$\begin{array}{l}\text { Geração de } \\
\text { Valor }\end{array}$} & $\begin{array}{l}\text { Agente de integração, } \\
\text { transformação e } \\
\text { impacto positivo na } \\
\text { sociedade }\end{array}$ & Número de inscrições em prêmios & $\begin{array}{l}\text { Número de pessoas de fora da } \\
\text { UFES participando dos } \\
\text { projetos do LabTAR }\end{array}$ & 1 & 1 & 30 & 0 & 0 & 14 & $0 \%$ & $0 \%$ & $47 \%$ \\
\hline & \multirow{2}{*}{$\begin{array}{c}\text { Referência em pesquisa } \\
\text { nas áreas de } \\
\text { competência }\end{array}$} & $\begin{array}{l}\text { Número de projetos de inovação e de } \\
\text { orientação em andamento }\end{array}$ & & 8 & 8 & & 8 & 8 & & \multirow[t]{2}{*}{$50 \%$} & \multirow[t]{2}{*}{$50 \%$} & \\
\hline & & Fator de impacto das publicações do LabTAR & & & & & & & & & & \\
\hline \multirow{6}{*}{ Stakeholders } & \multirow{3}{*}{$\begin{array}{l}\text { Divulgação e } \\
\text { publicidade }\end{array}$} & Número de projetos de divulgação & & 1 & 1 & 1 & 1 & 1 & 1 & \multirow{3}{*}{$67 \%$} & \multirow{3}{*}{$67 \%$} & \multirow{3}{*}{$33 \%$} \\
\hline & & Número de publicações no site & & 2 & 2 & 2 & 2 & 2 & 0 & & & \\
\hline & & Número de visitas ao site & & & & & & & & & & \\
\hline & $\begin{array}{l}\text { Publicações e } \\
\text { apresentações }\end{array}$ & Número de publicações e apresentações relacior & nadas às áreas de competência & 2 & 2 & 3 & 1 & 1 & 1 & $50 \%$ & $50 \%$ & $33 \%$ \\
\hline & $\begin{array}{l}\text { Atingir resultados } \\
\text { relevantes }\end{array}$ & Número de convites para parcerias e redes & & 1 & 1 & 1 & 1 & 1 & 2 & $100 \%$ & $100 \%$ & $100 \%$ \\
\hline & $\begin{array}{c}\text { Envolvimento em redes } \\
\text { de colaboração }\end{array}$ & Número de participações em redes de colaboraç & & 3 & 3 & 4 & 4 & 3 & 3 & $100 \%$ & $100 \%$ & $75 \%$ \\
\hline \multirow{9}{*}{$\begin{array}{l}\text { Processos } \\
\text { Internos }\end{array}$} & $\begin{array}{l}\text { Gerenciamento de } \\
\text { projetos }\end{array}$ & Nota de Gerenciamento de Projetos & & $75 \%$ & $85 \%$ & $85 \%$ & $71 \%$ & $73 \%$ & $79 \%$ & $95 \%$ & $86 \%$ & $93 \%$ \\
\hline & Gestão LabTAR & Nota do acompanhamento do BSC & & $90 \%$ & $95 \%$ & & $64 \%$ & $69 \%$ & & $71 \%$ & $73 \%$ & \\
\hline & \multirow{4}{*}{$\begin{array}{l}\text { Autossuficiência de } \\
\text { recursos }\end{array}$} & Número de Bolsistas & & 5 & 5 & 8 & 9 & 8 & 6 & \multirow{4}{*}{$82 \%$} & \multirow{4}{*}{$76 \%$} & \multirow{4}{*}{$74 \%$} \\
\hline & & \begin{tabular}{|l|} 
Número de Voluntários \\
\end{tabular} & & 5 & 5 & 10 & 3 & 1 & 5 & & & \\
\hline & & Número de Professores nas áreas de conhecime & & 6 & 6 & 6 & 4 & 5 & 5 & & & \\
\hline & & Índice de adequação de infraestrutura & & $90 \%$ & $90 \%$ & $90 \%$ & $95 \%$ & $90 \%$ & $80 \%$ & & & \\
\hline & Tecnologias inovadoras & Número de projetos de inovação em andamento & & 2 & 2 & 2 & 2 & 2 & 2 & $100 \%$ & $100 \%$ & $100 \%$ \\
\hline & \multirow{2}{*}{ Comunicação interna } & Percentual de presença em reuniões & & $70 \%$ & $70 \%$ & $70 \%$ & $50 \%$ & $65 \%$ & $71 \%$ & \multirow{2}{*}{$45 \%$} & \multirow{2}{*}{$65 \%$} & \multirow{2}{*}{$78 \%$} \\
\hline & & Percentual de leitura de atas & & $80 \%$ & $80 \%$ & $80 \%$ & $15 \%$ & $30 \%$ & $44 \%$ & & & \\
\hline \multirow{4}{*}{ Colaboradores } & \multirow{2}{*}{$\begin{array}{l}\text { Atrair e reter os } \\
\text { melhores }\end{array}$} & Percentual de permanência dos membros em fur & nção do tempo esperado & $80 \%$ & $80 \%$ & $80 \%$ & $100 \%$ & $50 \%$ & $82 \%$ & \multirow{2}{*}{$100 \%$} & \multirow{2}{*}{$81 \%$} & \multirow{2}{*}{$50 \%$} \\
\hline & & Percentual de membros novos com padrinho & & $100 \%$ & $100 \%$ & $100 \%$ & $100 \%$ & $100 \%$ & $0 \%$ & & & \\
\hline & $\begin{array}{l}\text { Compartilhamento de } \\
\text { conhecimentos }\end{array}$ & Número de Seminários & & 3 & 4 & 4 & 0 & 3 & 7 & $0 \%$ & $75 \%$ & $100 \%$ \\
\hline & Clima Organizacional & Resultado da Avaliação de Clima & & $85 \%$ & $85 \%$ & $85 \%$ & $77 \%$ & $77 \%$ & $77 \%$ & $91 \%$ & $91 \%$ & $91 \%$ \\
\hline
\end{tabular}

Quadro 2 - Balanced Scorecard do LabTAR ciclo 2012/2013 
Balanced Scorecard na Prática da Gestão de um Laboratório Acadêmico

\begin{tabular}{|c|c|c|c|c|}
\hline Perspectiva & Objetivo Estratégico & Indicadores & Meta & Iniciativas \\
\hline \multirow{3}{*}{ 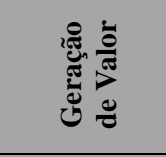 } & \multirow{2}{*}{ Geração de conhecimento } & $\mathrm{N}^{\circ}$ de convites para compartilhar conhecimentos do LabTAR & 1 & \\
\hline & & $\mathrm{N}^{0}$ de citações & 3 & \\
\hline & $\begin{array}{l}\text { Ser agente de integração de redes de } \\
\text { inovação eficazes }\end{array}$ & $\begin{array}{l}\mathrm{N}^{0} \text { de projetos que envolvem agentes de fora da comunidade } \\
\text { acadêmica }\end{array}$ & 3 & \\
\hline \multirow{5}{*}{ 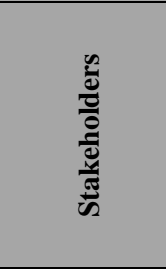 } & Publicações & Índice ponderado de publicações & & Levantar as publicações de interesse \\
\hline & $\begin{array}{lllll}\begin{array}{l}\text { Participar efetivamente } \\
\text { colaboração }\end{array} & \text { de } & \text { redes } & \text { de } \\
\end{array}$ & $\mathrm{N}^{\mathrm{o}}$ de projetos em rede em que o LabTAR participa & 3 & \\
\hline & \multirow{2}{*}{ Atender à comunidade com projetos } & $\mathrm{N}^{\circ}$ de projetos vinculados à comunidade & 1 & \\
\hline & & Índice de satisfação da comunidade com projetos & $60 \%$ & \\
\hline & $\begin{array}{l}\text { Desenvolver tecnologias, produtos e serviços } \\
\text { de apoio às redes de colaboração }\end{array}$ & $\mathrm{N}^{0}$ de novas tecnologias em desenvolvimento e desenvolvidas & 2 & Checar editais e passar informações para o informativo \\
\hline \multirow{11}{*}{ 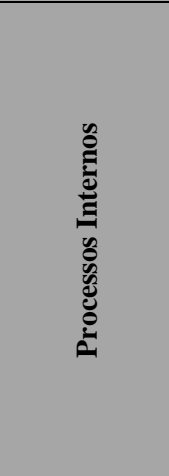 } & \multirow{2}{*}{ Prospecção de parcerias } & $\mathrm{N}^{\mathrm{o}}$ de parceiros levantados & & \multirow{2}{*}{ Conhecer os potenciais parceiros } \\
\hline & & $\mathrm{N}^{\circ}$ de parcerias realizadas & & \\
\hline & \multirow{2}{*}{ Comunicação com stakeholders } & \multirow{2}{*}{$\%$ de cumprimento do Plano de Comunicação com Stakeholders } & & Comunicação frequente com os stakeholders \\
\hline & & & & Informativo para os stakeholders \\
\hline & \multirow{4}{*}{ Comunicação interna eficaz } & \multirow{2}{*}{ Índice de satisfação com comunicação interna } & \multirow{2}{*}{$75 \%$} & Organização dos arquivos \\
\hline & & & & Criação do Site \\
\hline & & $\%$ de presença nas reuniões & $75 \%$ & \\
\hline & & \% leitura de atas de reunião & $95 \%$ & Plano de comunicação \\
\hline & \multirow{2}{*}{ Gerenciamento de projetos } & $\mathrm{N}^{\circ}$ de projetos entregues no prazo & $100 \%$ & \multirow{2}{*}{$\begin{array}{l}\text { Olhar editais (FAPES e CNPq) e passar informações } \\
\text { para o informativo }\end{array}$} \\
\hline & & Índice de gerenciamento (pesquisa) & $80 \%$ & \\
\hline & Autossuficiência de recursos & Índice de autossuficiência & $90 \%$ & Alavancar fontes de recursos próprios \\
\hline \multirow{9}{*}{ 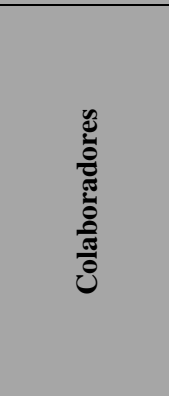 } & \multirow{2}{*}{ Clima organizacional } & Índice de pesquisa de Clima & $80 \%$ & \multirow{2}{*}{ Organizar LabTAR Aventura } \\
\hline & & Eventos de Integração & 3 & \\
\hline & \multirow{4}{*}{ Capacitação dos membros } & \multirow{2}{*}{$\mathrm{N}^{\circ}$ de seminários } & \multirow{2}{*}{3} & Organizar mini apresentações semanais \\
\hline & & & & Seminários internos e workshops \\
\hline & & $\%$ de cumprimento do check-list do plano de membro novo & $100 \%$ & Equipe no desafio Sebrae \\
\hline & & \% de participação nos seminários & $70 \%$ & Manual do membro novo e apadrinhamento \\
\hline & \multirow{2}{*}{ Atração e retenção de membros } & Tempo médio de ociosidade das bolsas & 2 & \multirow{3}{*}{ Organização dos arquivos } \\
\hline & & $\%$ de permanência dos membros & $100 \%$ & \\
\hline & $\begin{array}{l}\text { Crescimento pessoal e profissional dos } \\
\text { membros }\end{array}$ & İndice pessoal de crescimento & $70 \%$ & \\
\hline
\end{tabular}

Quadro 3 - Balanced Scorecard do LabTAR ciclo 2014 
Dentre as quatro perspectivas do BSC, a que mais sofreu alterações entre as versões foi a de Stakeholders. As mudanças foram motivadas pela percepção, por parte dos membros, de que os OE desta perspectiva necessitavam de reformulação, uma vez que não correspondiam à realidade. Essa percepção de desalinhamento com a realidade gerou uma queda no resultado dos indicadores, já que não havia incentivo para cumprir metas não alinhadas com a realidade do laboratório. O OE Atingir Resultados Relevantes foi transformando em Atender à Comunidade com Projetos, refletindo melhor especificação de quais resultados o LabTAR pretende atingir. Criou-se um OE específico para o desenvolvimento de tecnologias de apoio a redes de colaboração, algo que está no próprio nome do laboratório.

Vale ressaltar também que todos os OE que tiveram ao menos uma iniciativa completamente executada obtiveram uma evolução positiva em suas medições, enquanto a maioria dos objetivos que não tiveram suas iniciativas executadas obtiveram queda em suas medições, indicando coerência entre iniciativas e indicadores selecionados para cada OE.

\section{Relações causais}

Um diferencial do BSC apontado pelos seus proponentes é o estabelecimento de relações de causa e efeito entre OE e avaliação de sua validade com o sistema de medições. Entretanto, o aprendizado sobre a estratégia organizacional através das relações de causa e efeito expressas no BSC é demorado. No caso do BSC do LabTAR, poucas relações causais entre OE foram estabelecidas no $\mathrm{PE}$ inicial e, à medida que $\mathrm{o}$ ME e OE eram alterados, mais setas que representavam essas relações eram perdidas. Durante o processo, não houve ênfase em utilizar as medições para verificar as relações causais estabelecidas.

Na reunião de análise crítica, foi observado que o BSC inicial apresentava problemas nas relações de causa e efeito ou de indicadores e metas, ou ainda de defasagem no tempo dos resultados obtidos, dado que os resultados positivos obtidos nas Perspectivas de Stakeholders, Processos Internos e Colaboradores, não se refletiram na Geração de Valor, que teve desempenho fraco em todo o período.

O aprendizado estratégico a partir da verificação das hipóteses de causa e efeito entre $\mathrm{OE}$ não foi possível durante o período inicial de implantação do BSC no LabTAR. Esse resultado confirma a necessidade de tempo maior de amadurecimento do seu uso para ser possível a avaliação das relações causais no Mapa Estratégico.

\section{CONCLUSÕES}

A participação de todos os membros da organização, no caso um laboratório acadêmico, na construção dos pilares do Planejamento Estratégico, ou seja, na definição de Missão, Visão e Valores do LabTAR, foi fundamental. Ela contribuiu para a criação da identidade do grupo dando início à formação da cultura organizacional e aumentou o interesse dos membros em participar de outras atividades do laboratório além de suas atribuições específicas.

A Análise SWOT contribuiu para nivelar os conhecimentos do grupo sobre o ambiente no qual o LabTAR se encontra. Embora não tenha contribuído diretamente para a construção do ME, serviu de base para checar a coerência do ME com a realidade percebida.

A atividade de construção do ME foi modificada na versão 2014. Na elaboração da primeira versão os membros foram divididos em dois grupos para construir separadamente um ME cada. Em seguida, os grupos reuniram-se para a criação de um ME único. $\mathrm{Na}$ segunda rodada, o ME foi construído de uma vez por todo o grupo, o que se mostrou melhor, pois a participação em duas discussões para o mesmo fim gerou desinteresse de membros do grupo na elaboração do ME final.

Tanto a definição dos indicadores quanto das metas, embora feitas de forma colaborativa e com participação de todos, contaram apenas com bom senso e experiência prévia de alguns membros. Uma apresentação do assunto enriquecida com exemplos foi apontada como necessária na reunião de análise crítica feita ao final do processo.

A proposição e seleção das iniciativas feitas em conjunto favoreceu o engajamento do grupo, que teve a oportunidade de sugerir melhorias em todas as áreas dentro do laboratório. A atribuição de um responsável por cada iniciativa não foi suficiente para garantir a elaboração e execução dos planos de ação. O acompanhamento da coordenação sobre o andamento das ações é necessário bem como uma reavaliação da validade das iniciativas ao longo do processo, o que pode ser feito durante as reuniões de apresentação dos resultados de desempenho.

As relações de causa e efeito entre $\mathrm{OE}$ não foram valorizadas pelo grupo no processo. No entanto, a identificação e apreciação destas relações e seus impactos sobre o desempenho da organização são indispensáveis para o aprendizado estratégico. Desta maneira, pode-se afirmar que o BSC, no caso do LabTAR em seu primeiro período de uso, foi utilizado como um sistema de medição e ferramenta de comunicação visual, não cumprindo o seu papel de sistema de gestão estratégica. 
As relações de causa e efeito entre OE, declaradas como um dos principais diferenciais do BSC, não se mostraram relevantes nas medições do desempenho. $\mathrm{Na}$ construção do primeiro $\mathrm{ME}$, foram definidas poucas dessas relações e, ao longo das revisões quando três $\mathrm{OE}$ foram retirados, o número caiu ainda mais. A preocupação com o estabelecimento de maior número dessas relações na revisão do $\mathrm{ME}$ resultou em treze relações de causa e efeito estabelecidas, ligando todos os OE do BSC.

A importância da realização das iniciativas para o atingimento da Visão da organização fez-se clara dado que todos os $\mathrm{OE}$ que tiveram ao menos uma iniciativa completamente executada obtiveram uma evolução positiva em suas medições ao longo do tempo, enquanto a maioria dos objetivos que não tiveram suas iniciativas executadas obteve uma evolução negativa nos resultados ao longo das medições.

O PE deve ser constantemente revisto para adequar-se à realidade da organização. Ele deve adaptar-se às mudanças tanto no ambiente interno quanto no externo. Os responsáveis pelo controle e monitoramento da execução da estratégia também devem evoluir constantemente para acompanhar tais mudanças. O BSC, enquanto ferramenta de suporte ao $\mathrm{PE}$, é flexível o suficiente para adaptar-se a variados tipos de organização, de empresas a OSFL como é o caso do LabTAR.

Conclui-se, portanto, que o Balanced Scorecard, com suas devidas adaptações, é uma ferramenta que pode ser usada com sucesso na prática da gestão de um laboratório acadêmico. Em seus estágios iniciais de maturidade serve muito bem como ferramenta de comunicação. Sua eficácia como sistema de medição de desempenho depende de um nível de amadurecimento maior. Seu valor como sistema de gestão estratégica, entretanto, requer maiores níveis de aprendizagem e amadurecimento do que foi conseguido em pouco mais de um ano no caso estudado.

Laboratórios acadêmicos, por sua natureza, tendem a ter alta rotatividade de pessoas. $\mathrm{O}$ estabelecimento de uma cultura organizacional baseada nos fundamentos de missão, visão e valores e em sistemas de gestão que permitam desdobrar esses fundamentos em iniciativas e tarefas práticas do dia-adia como o BSC podem significar ganhos de perenidade para os laboratórios.

\section{REFERÊNCIAS}

Ackoff, R. L. (1970). A concept of corporate planning. New York: Wiley-Interscience.

Ansoff, H. I. (1965). Corporate strategy: an analytic approach to business policy for growth and expansion (p. 241). McGraw-Hill.

Bain, Rigby, D., \& Bilodeau, B. (2013). Management Tools \& Trends 2013.

Brignall, S. (2002). The unbalanced scoredcard: a social and environmental critique. Perfornance Measurement and Management: Research and Action, 85-92.

Hoque, Z. (2013). 20 years of studies on the balanced scorecard: Trends, accomplishments, gaps and opportunities for future research. The British Accounting Review, 1-27. doi:10.1016/j.bar.2013.10.003

Idenburg, P. J. (1993). Four styles of strategy development. Long Range Planning, 26(6), 132137.

Kaplan, R., \& Norton, D. (1992). The Balanced Scorecard - Measures that Drive Performance. Harvard Business Review, 70, 71-79. doi:00178012

Kaplan, R., \& Norton, D. (1993). Putting the balanced scorecard to work. Harvard Business Review, 71, 134-147. doi:10.1177/1056492604268208

Kaplan, R. S., \& Norton, D. P. (1996a). The Balanced Scorecard: Translating Strategy into Action (p. 336). Harvard Business Review Press.

Kaplan, R. S., \& Norton, D. P. (1996b). Using the Balanced Scorecard as a Strategic Management System. Harvard Business Review, 74, 75-85. doi:10.1016/S0840-4704(10)60668-0

Mendes, P., Santos, A. C., Perna, F., \& Teixeira, M. R. (2012). The balanced scorecard as an integrated model applied to the Portuguese public service: a case study in the waste sector. Journal of Cleaner Production, 24, 20-29. doi:10.1016/j.jclepro.2011.11.007

Mintzberg, H. (1973). Strategy-making in three modes. California Management Review, winter, 44-55.

Mintzberg, H. (1987). Crafting strategy. Harvard Business Review, July-August, 66-75.

Mintzberg, H. (1990). The Design School: reconsidering the basic premises of strategic 
management. Strategic Management Journal, 11, 171-195.

Niven, P. R. (2007). Balanced Scorecard - Passo - A Passo: Elevando o Desempenho e Mantendo Resultados (p. 403). Qualitymark Ed.

Norreklit, H. (2000). The balance on the balanced scorecard a critical analysis of some of its assumptions. Management Accounting Research, 11(1), 65-88. doi:10.1006/mare.1999.0121
Oliveira, D. R. de P. (2011). Planejamento estrategico: conceitos, metodologia, praticas (29a . ed., p. 335$)$. Atlas.

Quinn, J. B. (1989). Strategic change, logical incrementalism. Sloan Management Review, 55-58.

Steiner, G. A. (1979). Strategic Planning: what every manager must know (p. 383). New York: The Free Press. 